\title{
Extra-articular rheumatoid arthritis
}

\author{
E. Marcucci, E. Bartoloni, A. Alunno, M.C. Leone, G. Cafaro, F. Luccioli, \\ V. Valentini, E. Valentini, G.M.C. La Paglia, A.F. Bonifacio, R. Gerli \\ Rheumatology Unit, Department of Medicine, University of Perugia, Italy
}

\begin{abstract}
SUMMARY
Rheumatoid arthritis (RA) is a chronic inflammatory disease that mainly affects the joints, though a consistent proportion of patients may also display extra articular manifestations (EAMs). From rheumatoid nodules to interstitial lung disease, from cardiovascular events to vasculitis, the spectrum of EAMs encompasses various conditions with different prognoses. EAMs may also occur as first RA manifestation, therefore the coordination with other health professionals, including general practitioners, is needed. The aim of this article is to provide an overview on EAMs in RA with particular focus on the recognised risk factors and the available recommendations for managing them, as well as comorbidities in RA patients.
\end{abstract}

Key words: Rheumatoid arthritis; Cardiovascular disease; Pulmonary manifestations.

Reumatismo, 2018; 70 (4): 212-224

\section{INTRODUCTION}

$\mathrm{R}$ heumatoid arthritis (RA) is one of the most prevalent chronic inflammatory diseases (1). It primarily involves the joints, but it should be considered a systemic disease, as different extra-articular manifestations (EAMs), including rheumatoid nodules, pulmonary involvement or vasculitis could be also present (1). In addition, the disease has been associated with high prevalence of systemic comorbidities. EAMs in RA have been reported with a frequency ranging from $18 \%$ to $41 \%$, this wide range being mainly due to study design and differences in the populations enrolled (2). In recent years, new therapeutic strategies have allowed a better overall control of disease activity with subsequent reduced risk of severe EAMs, such as rheumatoid vasculitis (RV) (2). Despite the improved outcome of RA patients and the reduced incidence of systemic EAMs, diagnostic and therapeutic strategies for these comorbidities still remain a challenge. The aim of this review is to provide an overview of EAMs in RA patients, focusing on recent findings on the pathogenesis and possible predisposing factors associated with specific organ involvement. Moreover, an additional aim is to focus on the pivotal role of rheumatologists in pursuing a holistic approach to these patients through health promotion, health education and specific disease prevention strategies.

\section{PATHOGENESIS AND PREDISPOSING FACTORS FOR EXTRA-ARTICULAR RHEUMATOID ARTHRITIS}

The pathogenesis of extra-articular RA involves both natural and acquired immunological mechanisms. High levels of circulating immune complexes (CICs) and complement (in particular $\mathrm{C} 4$ ) consumption have been demonstrated in patients with severe EAMs and have been shown to predict mortality in patients with RA-associated vasculitis. Moreover, vasculitis and higher prevalence of EAMs are mainly observed in RA patients with rheumatoid factor (RF) positivity, in particular at high titer (2). Intriguingly, the direct involvement of RF in the formation of complement activating CICs further highlights its key role in the 
pathogenesis of EAMs in RA. On the other hand, the role of anti-cyclic citrullinated protein (anti-CCP) antibodies (Abs) in the pathogenesis of extra-articular RA is still a matter of debate. It has been suggested that these Abs may be involved, in associations with several concomitant pathogenic mechanisms, in the induction of subclinical atherosclerotic damage in RA patients, thus supporting their contribution to cardiovascular disease (CVD) development (35). Recently, high titers of anti-CCP Abs, in particular anti-citrullinated histone $\mathrm{H} 2 \mathrm{~b}$ Abs, have been demonstrated to be significantly associated with higher coronary artery calcification scores in a cohort of RA patients without clinical CVD, thus further supporting the involvement of this class of Abs in the induction of atherosclerotic damage (6). Furthermore, a recent study evaluated the contribution of Abs against mutated citrullinated vimentin (anti-MCV) in EAMs in a cohort of 225 RA patients (7). In this study, no association was observed between anti-CCP or anti-MCV levels and presence of EAMs in the whole group of patients. However, a sub-analysis according to each single manifestation revealed that rheumatoid nodules were associated with positivity for RF, anti-CCP and anti-MCV, while these auto-Abs were not associated with Sjögren's syndrome (SS), chronic anemia or peripheral neuropathy. Moreover, the levels of anti-CCP Abs correlated with the score of the Health Assessment Questionnaire-Disability Index (HAQ-D). Interestingly, high levels of antiCCP Abs have been detected in the lungs of RA patients with bronchiectasis (8). In particular, it has been hypothesized that infectious triggers and other environmental factors may induce a persistent antigen stimulation which, in genetically predisposed subjects, may lead to the development of RA. Indeed, the majority of lung manifestations occur in the first 5 years of the disease and they precede the onset of joint involvement in 10-20\% of the cases. Interestingly, genetic predisposition seems to exert a dual role in the pathogenesis of EAMs. In this setting, the human leucocyte antigen (HLA) DRB1*04 subtype has been demonstrated to correlate with EAMs occurrence, especially in RA patients developing vasculitis and Felty syndrome, while this haplotype seems to be protective against interstitial lung disease (ILD) onset (9). Finally, male sex and tobacco smoking are well-recognized risk factors for extraarticular involvement (8).

\section{CARDIOVASCULAR INVOLVEMENT}

The leading cause of death among RA patients is CVD, with a risk $50 \%$ higher than that observed in the general population (10). In addition to CVD, a recent metaanalysis demonstrated a higher risk of cerebrovascular disease in RA patients, in particular in patients aged $<50$ years, with a twice-increased risk in comparison to the general population (11).

It has been suggested that RA and CVD may share a common genetic predisposition and that similar inflammatory and immune-mediated mechanisms contribute to their pathogenesis (10). In line with this evidence and in order to early identify patients at higher $\mathrm{CV}$ risk, the European League Against Rheumatism (EULAR) update on CVD risk management recently proposed CVD risk assessment in all RA patients by Systematic Coronary Risk Evaluation (SCORE) algorithm at least every 5 years in case of low CVD risk (SCORE $<5 \%$ ) and sooner in case of intermediate or high risk (SCORE $\geq 5 \%,<10 \%$ and $\geq 10 \%$, respectively) (8). However, the contribution of several concomitant inflammatory and immune factors other than traditional $\mathrm{CV}$ risk factors in the pathogenesis of $\mathrm{CV}$ involvement in RA suggests caution in the interpretation and employment of CVD risk algorithms (12). The direct contribution of the disease in the acceleration of atherosclerosis has been further demonstrated by recent evidence that systemic inflammation predicts progression of carotid intima-media thickness, a validated surrogate marker of subclinical atherosclerotic damage, in RA patients at 3 years of follow-up (10). On the basis of this evidence, the EULAR task force recommends screening for ca- 
rotid artery atherosclerosis in patients with moderate CVD risk according to SCORE charts (12).

In association with autoimmune and inflammatory mechanisms, traditional risk factors exert a pivotal role in the pathogenesis of CV damage in RA (13). Among these, an impaired cholesterol homeostasis resulting in reduced high-density lipoprotein cholesterol levels in active RA and a higher prevalence of metabolic syndrome and hypertension in RA patients in comparison to general population have been recently demonstrated to contribute significantly to higher CV risk in these patients $(14,15)$. In particular, hypertension represents an important predictor of CV events in RA patients. Chronic inflammation together with immune-mediated mechanisms has been demonstrated to affect blood pressure control negatively in patients with systemic autoimmune disease, contributing to induction and perpetuation of premature atherosclerosis (16).

Concerning the influence of RA treatments in reducing $\mathrm{CV}$ risk through their action on the inflammatory process, a recent metaanalysis has shown $21 \%$ reduction of $\mathrm{CV}$ events in patients treated with methotrexate (MTX) compared to non-users (17). In addition, hydroxychloroquine (HCQ) use has been demonstrated to reduce incident CVD significantly in these subjects (18). Despite their contribution to reducing inflammation, corticosteroid (GC) and non-steroidal anti-inflammatory drug (NSAID) use seem to increase CV risk (19). Finally, biologic therapy has been demonstrated to reduce potentially the $\mathrm{CV}$ risk in these patients. In this setting, data derived from systematic reviews demonstrated that anti-tumor necrosis factor- $\alpha$ (TNF- $\alpha)$ treatment is associated with a significant $\mathrm{CV}$ risk reduction in RA patients, in particular in responders to therapy (20).

In addition to the increased CVD risk, RA is associated with other specific cardiac manifestations such as pericarditis, myocardial disease, coronary vasculitis and valvular involvement. A systematic review and meta-analysis of case-control studies employing echocardiographic assessment of valvular and pericardial involvement in RA patients asymptomatic for cardiac disease, revealed specific disease-related cardiac structure involvement (21). In particular, pericardial effusion, valvular nodules, valvular thickening with isolated valvular insufficiency, aortic root alteration, structural (increased left ventricular mass) and functional (diastolic dysfunction) left ventricular changes emerged as the most common manifestations of heart structure involvement in RA. This specific heart involvement in RA, although often asymptomatic and termed as silent rheumatoid heart disease, has been associated with an increased risk of both heart failure and CV mortality (21).

\section{PULMONARY INVOLVEMENT}

The lung is another common site of extraarticular disease in RA and includes manifestations related to airways, parenchymal, vascular and pleural involvement. The reported prevalence of lung involvement in RA patients is about $50 \%$, but it could be highly variable due to different study designs, different populations and mainly because it is often asymptomatic and detectable only through imaging studies (22). ILD has a reported prevalence of $10-20 \%$ (23). Notably, its identification and management require the combination of histopathological, clinical and radiological examinations, thus often making the evaluation of pulmonary involvement in RA patients a diagnostic challenge. The most common ILD subtype in RA is usual interstitial pneumonia (UIP) followed by non-specific interstitial pneumonia, but even organizing pneumonia, desquamative interstitial pneumonia, lymphocytic interstitial pneumonia and diffuse alveolar damage have been documented (24). A recent systematic review identified male gender, older age, lower lung diffusion capacity for carbon monoxide (DLCO), UIP subtype and fibrosis extent as significant predictors of mortality in RA-ILD (25). In the evaluation and assessment of ILD in RA patients, it is very important to differentiate between diseaserelated lung involvement and pulmonary 
manifestations related to adverse effects of rheumatic therapies or infection occurrence in immunocompromised patients. Concerning airways disease, it affects from $39 \%$ to $60 \%$ of RA patients and it may manifest as bronchiectasis, bronchiolitis, airway hyperreactivity, cricoarytenoid arthritis in large airways as well as constrictive and obstructive bronchiolitis in small airways (26). Finally, pleural involvement may vary from pleural effusions, pleuritis, pleural thickening to empyema, pneumothorax and trapped lung syndrome (Table I). In particular, pleural effusion represents the most common feature of pleural involvement in RA. It is usually unilateral, asymptomatic and occurs several years after disease diagnosis.

Table I - Pulmonary manifestations in rheumatoid arthritis.

\begin{tabular}{|l|}
\hline Interstitial lung disease \\
\hline Usual interstitial pneumonia \\
\hline Nonspecific interstitial pneumonia \\
\hline Organizing pneumonia \\
\hline Lymphocytic interstitial pneumonia \\
\hline Acute interstitial pneumonia \\
\hline Airways disease \\
\hline Follicular bronchiolitis \\
\hline Obliterative bronchiolitis \\
\hline Bronchiectasis \\
\hline Cricoarytenoid arthritis \\
\hline Rheumatoid nodules \\
\hline Pleural disease \\
\hline Pleuritis \\
\hline Pleural effusion \\
\hline Pneumothorax \\
\hline Empyema \\
\hline Vascular disease \\
\hline Pulmonary hypertension \\
\hline Vasculitis \\
\hline Caplan syndrome \\
\hline Drug toxicity \\
\hline Infections \\
\hline Amyloidosis \\
\hline Fibrobullous disease \\
\hline
\end{tabular}

\section{CUTANEOUS INVOLVEMENT}

Cutaneous manifestations in RA patients may vary from rheumatoid nodules, which are the most typical skin findings usually characterizing patients with long-standing disease, to RV, granulomatous disorders and neutrophilic dermatoses. Rheumatoid nodules occur in $20-35 \%$ of RA patients and their presence has been demonstrated to correlate with disease severity and higher levels of RF (27).

Furthermore, Kaushik et al. (28) recently examined the association between rheumatoid nodules and CVD in more than 26,000 RA patients, detecting a higher incidence rate of all $\mathrm{CV}$ events in RA patients with subcutaneous nodules compared to patients without nodules, independently of the presence of traditional CV risk factors. Skin is the most frequent localization of RV and frequent manifestations are represented by periungual infarctions (also called Bywater's lesions), petechiae, purpura, or erythema elevatum diutinum, a rare form of vasculitis presenting with firm symmetrical red-brown or violaceous papules and nodules on the extensor surface of the extremities. Necrotizing vasculitis of medium size vessels usually leads to reticular purpura, gangrene, deep ulcers of lower extremities or internal organ infarctions. Neutrophilic dermatoses, including Sweet's syndrome, pyoderma gangrenosum, rheumatoid neutrophilic dermatosis and sub-corneal pustular dermatosis, represent adjunctive manifestations of cutaneous involvement in RA (29).

In this setting, however, it is important to consider that cutaneous manifestations in RA patients may represent the expression of adverse effects related to concomitant treatments.

A wide spectrum of skin manifestations, including lichenoid skin eruption related to penicillamine, HCQ, or TNF- $\alpha$ inhibitors, accelerated rheumatoid nodulosis associated with MTX, infusion reactions, leukocytoclastic vasculitis, psoriasiform eruptions and anti TNF- $\alpha$-induced cutaneous lupus erythematosus, have been described in these patients (30). 


\section{GASTROINTESTINAL INVOLVEMENT}

Gastrointestinal (GI) manifestations in RA can vary from mild forms, which are observed in the majority of patients, to severe disease. In this setting, intestinal RV represents the most severe expression of GI involvement, reported in $10-38 \%$ of the cases, and it is characterized by intestinal small vessel inflammation causing ischemic ulcers and perforations or by devastating big vessel vasculitis with consequent extensive bowel infarctions (2).

Amyloidosis represents a rare complication of RA-associated GI disease, affecting from $7 \%$ to $13 \%$ of subjects (31). Duodenum is the most common localization with symptoms characterized by abdominal pain, nausea, intractable diarrhea and malabsorption. GI small vessel vasculitis or amyloidosis may involve also esophageal tract causing organ dysmotility with consequent dysphagia, heartburn and atrophic gastritis, as reported in up to $60 \%$ of RA patients.

Liver involvement in RA is rarely associated with clinical manifestations. Increased serum alkaline phosphatase and gammaglutamyl transaminase levels are common and usually correlate with disease activity (32). RA-associated liver disease is usually mild and it needs to be differentiated from autoimmune hepatitis, with possible extra-intestinal manifestations resembling RA, and from drug-induced disease, mainly related to aspirin, sulfasalazine or MTX assumption. Felty syndrome may represent an adjunctive cause of hepatomegaly in RA patients. It is a rare disease-related manifestation, commonly associated with long-standing and more severe disease and it is characterized by splenomegaly and neutropenia. In about $70 \%$ of patients, this syndrome leads to hepatomegaly, abnormal liver function tests and portal hypertension due to different mechanisms such as portal fibrosis, increased splenic blood flow and nodular regenerative hyperplasia (33).

\section{NEUROLOGIC INVOLVEMENT}

Neurologic involvement includes a wide spectrum of central and peripheral nervous system syndromes as result of small vessel vasculitis, joint damage or drug toxicity.

Peripheral nervous system (PNS) involvement has been reported in about $20 \%$ of RA patients and includes entrapment neuropathies, mononeuritis multiplex, distal sensory neuropathy and sensorimotor neuropathy (34). Non-compressive neuropathies, such as mononeuritis multiplex and sensorimotor neuropathies are the result of vasa nervorum vasculitis leading to vascular ischemia with subsequent axonal degeneration and neuronal demyelination. On the other hand, compressive neuropathies, such as carpal tunnel syndrome, are associated with articular deformities causing nerve compression, paresthesia and pain. Furthermore, chronic synovitis and structural joint changes of the midfoot and foot are often associated with the development of Morton neuroma and tarsal tunnel syndrome, two frequent causes of foot pain in these patients. Central nervous system (CNS) involvement in RA patients includes a wide variety of manifestations, such as cervical myelopathy, cerebral vasculitis, meningitis, optical atrophy and formation of rheumatoid nodules. The most common manifestation of CNS involvement is cervical myelopathy due to atlanto-axial subluxation or sub-axial subluxation, reported in up to $40 \%$ of RA patients (35). This severe complication represents the consequence of chronic inflammation of the atlanto-axial synovial tissue and may manifest with different symptoms ranging from neck pain and occipital headache to sensory deficits, lower cranial nerve palsy or transient ischemic attacks. As previously reported, the differential diagnosis of neurological involvement in RA should take into account the wide range of neurological adverse reactions to drugs commonly employed in RA patients. Among these, high doses of GC therapy may associate with psychiatric manifestations and cognitive impairment, sulfasalazine and MTX with headache and peripheral neuropathy and, finally, anti-malarials have been associated with retinal damage occurrence and ototoxicity $(36,37)$. In recent years, the widespread use of biologic agents has been 
associated with higher incidence of severe neurologic manifestations in these patients, including Guillain-Barré syndrome, Miller Fisher syndrome, chronic inflammatory demyelinating polyneuropathy, multifocal motor neuropathy, mononeuropathy multiplex and axonal sensorimotor polyneuropathies (36). Finally, up to $40 \%$ of RA patients may manifest psychiatric symptoms such as anxiety and depression, with a prevalence that is similar to other long-standing diseases as result of chronic pain (38).

\section{OCULAR INVOLVEMENT}

Ocular manifestations represent a significant part of EAMs in RA patients and vary from episcleritis to retinal vasculitis. Dry eye, often associated with secondary SS, represents the most frequent manifestation of ocular involvement affecting one out of four patients and often complicated by chronic conjunctivitis and corneal ulcers (39).

Episcleritis is a mild ocular condition affecting RA patients with a prevalence of $0.2-3.7 \%$ (40). It is bilateral in $40 \%$ of cases and appears as a typical salmon pink vasodilation of episcleral radial vessels. Scleritis affects $0.2-6.3 \%$ of RA patients and it is associated with an increased disease severity and mortality rate, particularly the necrotizing form which manifests with severe ocular pain increased by eye movements. Peripheral ulcerative keratitis is a further ocular manifestation with a prevalence of $3 \%$ and could present as a form of scleritis, usually in subjects with severe and long-standing disease (41). Symptoms associated with this condition are pain and redness of the eye involving both eyes in $40 \%$ of cases. A recent study, analyzing the influence of anti-CCP autoAbs in RA-associated ocular manifestations, demonstrated a strong correlation between this Abs positivity and ocular involvement (42).

\section{RENAL INVOLVEMENT}

Renal involvement in RA can be due to the disease itself, to complications of therapy or, finally, may appear as an epiphenomenon of a long-standing inflammatory process. Renal adverse effects of therapies and secondary amyloidosis represent the most common causes of kidney involvement. On the other hand, renal involvement as direct result of the disease itself is less common and usually manifests as mesangial proliferative glomerulonephritis (GN) or membranoproliferative GN and, rarely, as focal or diffuse proliferative GN. Mesangial proliferative GN in RA may have either immunoglobulin (Ig) M or IgA predominant pattern on immunofluorescence (43). In a recent retrospective and observational study aimed at analyzing 67 renal biopsies of RA patients, the most common histopathologic finding was renal amyloidosis $(31 \%)$, followed by mesangial GN (18\%), membranous nephropathy (17\%), extra-capillary proliferative GN (15\%), focal segmental glomerular sclerosis (9\%), minimal change nephropathy $(5 \%)$ and tubulointerstitial nephritis (5\%) (44). Membranous nephropathy was related to the use of gold salts in $45 \%$ of cases and its frequency has decreased since 1990.

\section{RHEUMATOID VASCULITIS}

$\mathrm{RV}$ is an uncommon complication of RA affecting 2-3\% patients (2). It may involve small and medium-size arteries and is usually observed in long-standing and severe forms of the disease. The incidence of RV has been decreasing since the ' $90 \mathrm{~s}$, probably due to improved treatments for the disease (45). Cutaneous vasculitis is the most common manifestation of RV, but it can potentially involve all body sites, in particular PNS, arteries of fingers and toes, causing digital ischemia, and eyes with consequent scleritis. In addition, generalized symptoms such as fever and weight loss are common (Table II). In clinical practice, $\mathrm{RV}$ is considered when manifestations of vasculitis unexplained by other pathologic conditions such as diabetes, atherosclerosis, infection, drug hypersensitivity or malignancy occur in a patient with established diagnosis of RA. Other pathologic conditions, in fact, are characterized by a clinical presentation that could resemble RV, including atherosclero- 
Table II - Vasculitic manifestations in rheumatoid arthritis.

\begin{tabular}{|l|l|}
\hline Site & Manifestation \\
\hline Skin & - Ischemic focal digital lesions \\
& $\begin{array}{l}\text { - Purpura, petechiae, ulcers } \\
\text { - Pyoderma gangrenosum } \\
\text { - Erythema elevatum diutinum }\end{array}$ \\
\hline Peripheral nerves & $\begin{array}{l}\text { - Mononeuritis multiplex } \\
\text { - Sensory peripheral neuropathy }\end{array}$ \\
\hline Central nervous system & $\begin{array}{l}\text { - Seizures } \\
\text { - Confusional state } \\
\text { - Hemiparesis }\end{array}$ \\
\hline Eyes & - Peripheral ulcerative keratitis \\
\hline Heart & $\begin{array}{l}\text { - Pericarditis } \\
\text { - Coronary arteritis } \\
\text { - Arrhythmias }\end{array}$ \\
\hline Kidneys & $\begin{array}{l}\text { - Amyloidosis } \\
\text { - Glomerulonephritis }\end{array}$ \\
\hline Lung & $\begin{array}{l}\text { - Diffuse alveolar hemorrhage } \\
\text { - Pleuritis }\end{array}$ \\
\hline Gastrointestinal & - Ischemic bowel \\
\hline Systemic manifestations & $\begin{array}{l}\text { - Fever } \\
\text { - Weight loss }\end{array}$ \\
\hline
\end{tabular}

sis, venous insufficiency and infections. As a consequence, histological demonstration of vasculitis in the affected organs should be recommended in all patients with specific manifestations, even though it is not always feasible. In this case, surrogate criteria, such as the presence of classic ischemic skin lesions (except for isolated digital infarcts) or mononeuritis multiplex in a patient with established RA and no other possible explanations, are generally sufficient for a presumptive diagnosis (46). Finally, rare case reports described inflammatory involvement of large-size vessels (aortitis) in RA patients. In this setting, high-titers of RF, long-standing disease, erosive disease and rheumatoid nodules have been demonstrated to be significant risk factors for large vessel involvement in these patients (47).

\section{RHEUMATOID ARTHRITIS AND INFECTIONS}

Serious infections are a major concern in RA patients, being one of the three leading causes of death in this population (48). When evaluating the infection risk in RA, we need to take into account multiple predisposing factors, including disease activity, comorbidities and the use of immunosuppressive drugs (48). Indeed, disease severity and comorbidities, particularly chronic obstructive lung disease and chronic kidney diseases, are both main risk factors for serious infections, especially in older patients (49). Concerning the role of treatments, long-term GC therapy has been widely demonstrated to contribute to infections in patients with rheumatic and non-rheumatic diseases. In a meta-analysis of observational studies, a significantly increased risk for serious infections in RA patients treated with GC was demonstrated and the risk was dose-dependent, being markedly increased for subjects receiving up to $10 \mathrm{mg} /$ day of prednisone (50). In addition, cytokine inhibitors and other biological agents have been demonstrated to increase the risk of serious infections in several randomised clinical trials and observational cohort studies (51). In particular, the risk seems to be higher within the first six months of therapy and to decline over time (52).

The most common sites of serious infections are the lower respiratory tract followed by skin and soft tissues (53). Singh et al. (54) have recently conducted a systematic review including both traditional and meta-analysis in order to assess the risk of serious infections in RA patients treated with biologic therapy in comparison to traditional disease-modifying anti-rheumatic drugs (DMARDs). Standard and high-dose biologics were associated with higher risk of serious infections compared to traditional DMARDs, while low doses were not. Furthermore, the risk was lower in patients who were methotrexate naïve compared with patients treated with traditional DMARDs or anti-TNF- $\alpha$.

In order to provide an estimation of serious infection risk in these patients, a risk score for serious infections has been developed in the German Rheumatoid Arthritis Observation of Biologic Therapy (RABBIT) register. The score includes age, measure of disability, comorbidities (chronic lung 
disease and renal failure), history of previous infections, previous treatment failure and current treatment (55).

RA subjects with a high-risk score had a 30-fold increased risk of developing serious infections and validation in a separate cohort demonstrated high agreement between observed and expected rates of serious infections $(56,57)$.

\section{RHEUMATOID ARTHRITIS AND MALIGNANCIES}

The overall incidence of malignancies in RA does not appear to be substantially higher than the risk expected in the general population (58). However, an increased rate of lymphoma, both Hodgkin's and non-Hodgkin's lymphoma, has been clearly recognized in RA patients in comparison to the general population (58). In particular, an association with diffuse large B-cell lymphoma has been reported in these patients, representing up to two-thirds of the lymphomas in RA patients (59). The risk of developing lymphoma is particularly increased in patients with high and longstanding disease activity, thus reflecting the role of chronic activation of $\mathrm{B}$ cells in the pathogenesis of lymphoproliferative disorders in RA as in other autoimmune conditions (60). An increased risk of lung cancer in RA patients has been documented in several studies (61).

This may be due to chronic lung inflammation linked to the disease itself in association with the smoking habit. On the contrary, a decreased risk of colorectal cancer has been observed in RA patients, partly attributable to long-standing treatment with NSAID (58).

Reduced rates of other cancer types, including breast, ovary, endometrial and prostate cancer have been reported in some studies (62). Finally, concerning the potential role of biologic therapies in malignancies development, many randomized controlled trials have shown no statistically significant increased risk of any type of cancer with biologic therapy versus controls (63). However, recent data derived from a Swedish biologics registry reported a 50\% in- creased risk of melanoma in patients treated with biologic therapy (64).

\section{RHEUMATOID ARTHRITIS AND BONE INVOLVEMENT}

RA represents the prototype disease of disrupted bone homeostasis. Loss of articular (erosions) and periarticular bone (demineralization) is the result of excessive bone resorption associated with highly limited bone formation (65). It has been postulated that the inflammatory burden characterizing disease pathogenesis may increase osteoclastogenesis, reducing osteoblastogenesis. In particular, recent studies have shown that several cytokines, such as TNF- $\alpha$, interleukin (IL)-1, IL-6, IL-17 and immune cells, have osteoclastogenic effects, mainly through the upregulation of the receptor activator of the nuclear factor kappa B ligand (RANKL) expression (66). These pro-inflammatory cytokines negatively impact osteoblast differentiation and their ability to produce mineralized matrix (67). Moreover, recent evidence suggests that multiple immune-mediated pathways may promote bone changes in RA patients. In this setting, anti-CCP and RF have been demonstrated to exert a pivotal role in mediating bone loss, not only by promoting the macrophage production of TNF- $\alpha$, but also through direct interaction with osteoclast precursors (68). This finding is supported by the observation that autoantibody positive patients have lower bone mass already at disease onset and that they are characterized by more severe bone damage during disease course in comparison to seronegative RA patients (69).

In addition to anti-CCP and RF, anti-acetylated and carbamylated proteins (anti-CarP) antibodies, identified in a small proportion of anti-CCP-negative RA patients, are associated with more severe radiographic progression (70). However, the systemic inflammatory process and immune system dysregulation are not the only factors responsible for bone loss and increased fracture risk. Indeed, GC intake and physical inactivity need to be taken into account in this population (71). In the Consortium 
of Rheumatology Researchers of North America (CORRONA) registry, which includes 8,419 female RA patients, postmenopausal status, higher modified Health Assessment Questionnaire (mHAQ) score and GC use were associated with higher fracture risk (72). Many targets have been identified for therapeutic intervention in order to restore the balance between bone loss and bone formation. Among these, denosumab, an anti-RANKL antibody, has been demonstrated to slow RA erosive process and to restore bone mineral density in clinical trials (73).

Furthermore, the use of TNF- $\alpha$ inhibitors was associated with a reduction in systemic and local bone loss, further confirming the key role of systemic inflammation in osteoporosis development. However, evidence on their effect in fracture prevention is still lacking (74). In conclusion, given the increased risk of osteoporosis in RA patients and its potential adverse impact on the quality of life and life expectancy, it is important for this population to undergo regular monitoring of bone mineral den- sity and to receive calcium and vitamin D supplementation and preventive therapy as needed.

\section{TREATMENT OF EXTRA ARTICULAR MANIFESTATIONS}

Both incidence and impact of EAMs in RA patients is now lower than previously reported, thanks to a better pharmacological control of disease activity. The management of EAMs requires early identification of specific manifestations and the introduction of targeted treatment according to their severity (Table III). With specific regard to ILD treatment, the evidence is quite low, as there are no randomized controlled trials and the limited data come from series, case reports, or small-size trials. Higher age, low forced vital capacity (FVC) and decrease in DLCO over time seem to be predictors of poor outcome (75). Treatment goals in ILD are based on categorization of the disease by its behavior and take into consideration fibrosis extent on high resolution computed tomography (HRTC) and DLCO reduction.

Table III - Management of extra-articular manifestations in rheumatoid arthritis.

\begin{tabular}{|c|c|c|}
\hline $\begin{array}{l}\text { Affected tissue } \\
\text { or organ }\end{array}$ & Extra-articular manifestations & Treatment \\
\hline \multirow[t]{2}{*}{ Heart } & Pericarditis & $\mathrm{GC}$, pericardiocentesis \\
\hline & Myocarditis or endocarditis & GC \\
\hline \multirow{2}{*}{ Lung } & ILD & GC, cyclophosphamide, mycophenolate, azathioprine \\
\hline & Pleuritis & GC, thoracentesis \\
\hline \multirow[t]{3}{*}{ Skin } & Rheumatoid nodules & Local GC, no treatment \\
\hline & Cutaneous vasculitides & GC, MTX, colchicine, dapsone, RTX \\
\hline & Neutrophilic dermatoses & Topical GC, dapsone, HCQ, anti-TNF \\
\hline \multirow{2}{*}{$\begin{array}{l}\text { Gastrointestinal } \\
\text { system }\end{array}$} & Secondary amyloidosis & \multirow[t]{2}{*}{ GC, cyclophosphamide, mycophenolate, anti-TNF } \\
\hline & Vasculitis & \\
\hline \multirow[t]{3}{*}{ Nervous system } & Mononeuritis multiplex & \multirow[t]{3}{*}{ GC, cyclophosphamide } \\
\hline & Sensory peripheral neuropathy & \\
\hline & Central nervous system vasculitis & \\
\hline \multirow[t]{2}{*}{ Eyes } & Scleritis/episcleritis & Local GC, cyclosporine, MTX, anti-TNF, RTX \\
\hline & Retinal vasculitis & GC, cyclophosphamide \\
\hline \multirow[t]{3}{*}{ Kidney } & Secondary amyloidosis & \multirow{3}{*}{$\begin{array}{l}\text { GC, cyclophosphamide, mycophenolate, anti-TNF, } \\
\text { hemodialysis }\end{array}$} \\
\hline & Glomerulonephritis & \\
\hline & Interstitial nephritis & \\
\hline
\end{tabular}

MTX, methotrexate; RTX, rituximab; $\mathrm{HCQ}$, hydroxychloroquine; GC, glucocorticoids. 
In this setting, it is possible to distinguish between self-limited, reversible, stable or progressive disease. Treatment should be considered in case of extensive disease (extent of fibrosis on HRCT $>30 \%$, DLCO $<54 \%$, desaturation with exercise), deterioration (decrease from baseline in FVC by $10 \%$ or DLCO by $15 \%$ ) or if the patient is symptomatic (76). As previously reported, there are no randomized controlled trials for the management of RA-ILD, but there are reports of the efficacy of prednisolone/ azathioprine, prednisolone/cyclophosphamide, cyclophosphamide, azathioprine, HCQ, d-penicillamine, and cyclosporine $(77,78)$. In this context, rapidly progressive ILD and even other major organ involvement, such as RV, usually require high doses of GC and intravenous cyclophosphamide or rituximab, drugs which have been successfully employed as either first and second line or rescue therapy (79) Concerning the role of anti-TNF- $\alpha$ in RV management, there is still ongoing debate about their function in RV prevention or treatment, as no prospective trials have been performed (2). Experience with randomized trials in other forms of vasculitis has shown inefficacy of TNF- $\alpha$ inhibition in these conditions, while in a retrospective chart review published in 2008, complete remission using these drugs was achieved in five out of nine cases $(56 \%)$ of RV refractory to conventional immunosuppressive therapy (80).

In addition to pharmacologic therapy, management of comorbidities (such as osteoporosis and fibromyalgia), promotion of health (smoking cessation and physical exercise) and coordination of integrative care in these patients with multiple comorbidities surely play a pivotal role in the management of EAMs (81).

\section{CONCLUSIONS}

Extra-articular RA can involve multiple tissues and organs and cause comorbidities and psychosocial consequences. It remains, therefore, a major diagnostic and therapeutic challenge in several patients. Even though a better overall control of disease activity has been associated with reduced risk for EAMs, no randomized controlled trials are currently available for managing major organ involvement such as rapidly progressive ILD or RV. In these pathologic conditions, treatment with cyclophosphamide and high-dose GC is recommended.

Alongside investigations on pathogenic mechanisms underlying RA, proper management is crucial of established risk factors for specific organ involvement in these patients, such as hypertension, dyslipidemia, insulin resistance, diabetes, obesity and cigarette smoking. The available recommendations for managing EAMs and comorbidities in RA patients reflect the need for a holistic approach to the process of care and treatment of adverse events, the encouragement of coordination with other health professionals, including general practitioners and, finally, the need to educate patients, not only concerning their disease and targeted treatments, but also with regard to health promotion and specific preventive actions.

\section{REFERENCES}

1. Smolen JS, Aletaha D, McInnes IB. Rheumatoid arthritis. Lancet. 2016; 388: 2023-38.

2. Kishore S, Maher L, Vikas M. Rheumatoid vasculitis: a diminishing yet devastating menace. Curr Rheumatol Rep. 2017; 19: 39.

3. Bartoloni Bocci E, Shoenfel Y, Gerli R. Inflammatory and autoimmune mechanisms in the induction of atherosclerotic damage in systemic rheumatic diseases: two faces of the same coin. Arthritis Care Res. 2011; 63: 178-83.

4. Gerli R, Vaudo G, Bartoloni Bocci E, et al. Different roles for anti- cyclic citrullinated peptide antibodies and CD4+CD28 null cells in the acceleration of atherosclerosis in rheumatoid arthritis: comment on the article by Farragher et al. Arthritis Rheum. 2009; 60: 631-2.

5. Gerli R, Bartoloni Bocci E, Sherer Y, et al. Association of anti-cyclic citrullinated peptide antibodies with subclinical atherosclerosis in patients with rheumatoid arthritis. Ann Rheum Dis. 2008; 67: 724-5.

6. Geraldino-Pardilla L, Giles JT, Sokolove J, et al. Association of anti-citrullinated peptide antibodies with coronary artery calcification in rheumatoid arthritis. Arthritis Care Res. 2017; 69: 1276-81. 
7. Gonzalez-Lopez L, Rocha-Muñoz AD, PonceGuarneros M, et al. Anti-cyclic citrullinated peptide (anti-CCP) and anti-mutated citrullinated vimentin (anti-MCV) - Relation with extra-articular manifestations in rheumatoid arthritis. J Immunol Res. 2014; 2014: 1-10.

8. Cafaro G, Alunno A, Valentini V, et al. The onset site of rheumatoid arthritis: the joints or the lung? Reumatismo. 2016; 68: 167-75.

9. Furukawa H, Oka S, Shimada K, et al. Association of human leukocyte antigen with interstitial lung disease in rheumatoid arthritis: a protective role for shared epitope. PLoS One. 2012; 7: e33133.

10. Choy E, Ganeshalingam K, Semb AG, et al. Cardiovascular risk in rheumatoid arthritis: recent advances in the understanding of the pivotal role of inflammation, risk predictors and the impact of treatment. Rheumatology. 2014; 53: 2143-54.

11. Wiseman SJ, Ralston SH, Wardlaw JM. Cerebrovascular disease in rheumatic diseases. A systematic review and meta-analysis. Stroke. 2016; 47: 943-50.

12. Agca R, Heslinga SC, Rollefstad S, et al. EULAR recommendations for cardiovascular disease risk management in patients with rheumatoid arthritis and other forms of inflammatory joint disorders: 2015/2016 update. Ann Rheum Dis. 2017; 76: 17-28.

13. Lauper K, Gabay C. Cardiovascular risk in patients with rheumatoid arthritis. Semin Immunopathol. 2017; 39: 447-59.

14. Charles-Schoeman C, Lee YY, Grijalva V, et al. Cholesterol efflux by high density lipoproteins is impaired in patients with active rheumatoid arthritis. Ann Rheum Dis. 2014; 71: 1157-62.

15. Kerekes G, Nurmohamed MT, Gonzalez-Gay MA, et al. Rheumatoid arthritis and metabolic syndrome. Nat Rev Rheumatol. 2014; 10: 691-6.

16. Bartoloni E, Alunno A, Gerli R. Hypertension as a cardiovascular risk factor in autoimmune rheumatic diseases. Nat Rev Cardiol. 2018; 15: 33-44.

17. Micha R, Imamura F, von Ballmoos MW, et al. Systematic review and meta-analysis of methotrexate use and risk of cardiovascular disease. Am J Cardiol. 2011; 108: 1362-70.

18. Sharma TS, Wasko MC, Tang X, et al. Hydroxychloroquine use is associated with decreased incident cardiovascular events in Rheumatoid Arthritis patients. J Am Heart Assoc. 2016 [epub ahead of print].

19. Mahdi M, Saeed A, Reza F, et al. New insights to the mechanisms underlying atherosclerosis in rheumatoid arthritis. Int J Rheum Dis. 2017; 20: 287-97.

20. Westlake SL, Colebatch AN, Baird J, et al. Tumour necrosis factor antagonists and the risk of cardiovascular disease in patients with rheumatoid arthritis: a systematic literature review. Rheumatology (Oxford). 2011; 50: 518-31.

21. Corrao S, Messina S, Pistone G, et al. Heart involvement in Rheumatoid Arthritis: Systematic review and meta-analysis. Int $\mathrm{J}$ Cardiol. 2013; 167: 2031-8.

22. O'Dwyer DN, Armstrong ME, Cooke G, et al. Rheumatoid arthritis (RA) associated interstitial lung disease (ILD). Eur J Int Med. 2013; 24: 597-603.

23. Sihvonen S, Korpela M, Laippala $P$, et al. Death rates and causes of death in patients with rheumatoid arthritis: a population-based study. Scand J Rheumatol. 2004; 33: 221-7.

24. Clive K, Kundan I, Iman-Gutierrez L, et al. Lung involvement in inflammatory rheumatic diseases. Best Pract Res Clin Rheumatol. 2016; 30: 870-88.

25. Assayag D, Lubin M, Lee JS, et al. Predictors of mortality in rheumatoid arthritis related interstitial lung disease. Respirology. 2014; 19 : 493-500.

26. Scott DL, Wolfe F, Huizinga TWJ, et al. Rheumatoid arthritis. Lancet. 2010; 376: 1094-108.

27. Nyha"1l-Wahlin B-M, Turesson C, Jacobsson LTH, et al. The presence of rheumatoid nodules at early rheumatoid arthritis diagnosis is a sign of extraarticular disease and predicts radiographic progression of joint destruction over 5 years. Scand J Rheumatol. 2011; 40: 81-7.

28. Kaushik P, Solomon DH, Greenberg JD, et al. Subcutaneous nodules are associated with cardiovascular events in patients with rheumatoid arthritis: results from a large US registry. Clin Rheumatol. 2015; 34: 1697-704.

29. Chua-Aguilera CJ, Möller B, Yawalkar N. Skin manifestations of rheumatoid arthritis, juvenile idiopathic arthritis, and spondyloarthritides. Clin Rev Allergy Immunol. 2017 [epub ahead of print].

30. Xue Y, Cohen JM, Wright NA, et al. Skin signs of rheumatoid arthritis and its therapy-induced cutaneous side effects. Am J Clin Dermatol. 2016; 17: 147-62.

31. Turesson C. Extra-articular rheumatoid arthritis. Curr Opin Rheumatol. 2013; 25: 360-6.

32. Ohira H, Abe K, Takahashi A. Involvement of the liver in rheumatic diseases. Clin J Gastroenterol. 2012; 5: 9-14.

33. Ebert EC, Hagspiel KD. Gastrointestinal and hepatic manifestations of rheumatoid arthritis. Dig Dis Sci. 2011; 56: 295-302.

34. Agarwal V, Singh R, Wiclaf, et al. A clinical, electrophysiological, and pathological study of neuropathy in rheumatoid arthritis. Clin Rheumatol. 2008; 27: 841-4.

35. Younes M, Belghali S, Kriaa S, et al. Compared imaging of the rheumatoid cervical spine: prevalence study and associated factors. Jt Bone Spine. 2009; 76: 361-8. 
36. Ramos-Remus C, Duran-Barragan S, Ortiz JDC. Beyond the joints: neurological involvement in rheumatoid arthritis. Clin Rheumatol. 2012; 31:1-12.

37. Benyamin RM, Vallejo R, Kramer J, et al. Corticosteroid induced psychosis in the pain management setting. Pain Physician. 2008; 11: $917-20$

38. Joaquim AF, Appenzeller S. Neuropsychiatric manifestations in rheumatoid arthritis. Autoimmun Rev. 2015; 14: 1116-22.

39. Turesson C. Extra-articular rheumatoid arthritis. Curr Opinion Rheumatol. 2013; 25: 360-6.

40. Sainz de la Maza M, Molina N, GonzalezGonzalez LA, et al. Clinical characteristics of a large cohort of patients with scleritis and episcleritis. Ophthalmology. 2012; 119: 43-50.

41. Artifoni M, Rothschild PR, Brézin A, et al. Ocular inflammatory diseases associated with rheumatoid arthritis. Nat Rev Rheumatol. 2014; 10: 108-16.

42. Vignesh AP, Srinivasan R. Ocular manifestations of rheumatoid arthritis and their correlation with anti-cyclic citrullinated peptide antibodies. Clin Opthalmol. 2015; 9: 393-7.

43. Mittal T, Rathi M. Rheumatological diseases and kidneys: a nephrologist's perspective. Int J Rheum Dis. 2014; 17: 834-44.

44. Vinicki JP, Pellet SC, De Rosa G, et al. Analysis of 65 renal biopsies from patients with Rheumatoid Arthritis (1976-2015): change in treatment strategies decreased frequency and modified histopathological findings. J Clin Rheumatol. 2015; 21: 335-40.

45. Makola A, Mattesona EL, Warrington KJ. Rheumatoid vasculitis: an update. Curr Opin Rheumatol. 2015; 27: 63-70.

46. Genta MS, Genta RM, Gabay C, et al. Systemic rheumatoid vasculitis: a review. Semin Arthritis Rheum. 2006; 36: 88-98.

47. Kaneko S, Yamashita H, Sugimori Y, et al. Rheumatoid arthritis-associated aortitis: a case report and literature review. SpringerPlus. 2014; 3: 509.

48. Doran MF, Crowson CS, Pond GR, et al. Predictors of infections in rheumatoid arthritis. Arthritis Rheum. 2002; 46: 2294-300.

49. Cutolo M, Kitas GD, van Riel PL. Burden of disease in treated rheumatoid arthritis patients: going beyond the joint. Semin Arthritis Rheum. 2014; 43: 479-88.

50. Dixon WG, Suissa S, Hudson M. The association between systemic glucocorticoid therapy and the risk of infection in patients with rheumatoid arthritis: systematic review and metaanalyses. Arthritis Res Ther. 2011; 13: R139.

51. Martin-Mola E, Balsa A. Infectious complications of biologic agents. Rheum Dis Clin North Am. 2009; 35: 183-99.

52. Galloway JB, Hyrich KL, Mercer LK, et al. Anti-TNF therapy is associated with an in- creased risk of serious infections in patients with rheumatoid arthritis especially in the first six months of treatment: updated results from the British Society for Rheumatology Biologics Register with special emphasis on risks in the elderly. Rheumatology (Oxford). 2010; 50: 124-31.

53. Humphrey J, Hyrich K, Simmons D. What is the impact of biologic therapies on common co-morbidities in patients with rheumatoid arthritis? Arthrit Res Ther. 2016; 18: 282.

54. Singh JA, Cameron C, Noorbaloochi, et al. The risk of serious infection with biologics in treating patients with rheumatoid arthritis: a systematic review and meta-analysis. Lancet. 2015; 18: 258-65.

55. Strangfeld A, Eveslage M, Schneider M, et al. Treatment benefit or survival of the fittest: what drives the time-dependent decrease in serious infection rates under TNF inhibition and what does this imply for the individual patient? Ann Rheum Dis. 2011; 70: 1914-20.

56. Zink A, Manger B, Kaufmann J, et al. Evaluation of the RABBIT Risk Score for serious infections. Ann Rheum Dis. 2014; 73: 1673-6.

57. Turesson C. Comorbidity in rheumatoid arthritis. Swiss Med Wkly. 2016; 146: w1490.

58. Smitten AL, Simon TA, Hochberg MC, et al. A meta-analysis of the incidence of malignancy in adult patients with rheumatoid arthritis. Arthritis Res Ther. 2008; 10: R45.

59. Baecklund E, Sundstrom C, Ekbom A, et al. Lymphoma subtypes in patients with rheumatoid arthritis: increased proportion of diffuse large B cell lymphoma. Arthritis Rheum. 2003; 48: 1543-50.

60. Baecklund E, Iliadou A, Askling J, et al. Association of chronic inflammation, not its treatment, with increased lymphoma risk in rheumatoid arthritis. Arthritis Rheum. 2006; 54: 692-701.

61. Hellgren K, Smedby KE, Feltelius N, et al. Do rheumatoid arthritis and lymphoma share risk factors? A comparison of lymphoma and cancer risks before and after diagnosis of rheumatoid arthritis. Arthritis Rheum. 2010; 62: 1252-8.

62. Simon TA, Thompson A, Gandhi KK, et al. Incidence of malignancy in adult patients with rheumatoid arthritis. Arthritis Res Ther. 2015; 17: 212 .

63. Lopez-Olivo MA, Tayar JH, Martinez-Lopez $\mathrm{JA}$, et al. Risk of malignancies in patients with rheumatoid arthritis treated with biologic therapy: a meta-analysis. JAMA. 2012; 308: 898-908.

64. Raaschou P, Simard JF, Holmqvist M, et al. Rheumatoid arthritis, anti-tumor necrosis factor therapy, and risk of malignant melanoma: nationwide population based prospective co- 
hort study from Sweden. BMJ. 2013; 346: 1939.

65. Shim JH, Stavre Z, Gravallese EM. Bone loss in rheumatoid arthritis: basic mechanisms and clinical implications. Calcif Tissue Int. 2017 [epub ahead of print].

66. Jung SM, Kim KW, Yang CW, et al. Cytokinemediated bone destruction in rheumatoid arthritis. J Immunol Res. 2014; 2014: 263625.

67. Heinlen L, Humphrey MB. Skeletal complications of rheumatoid arthritis. Ostoeoporos Int. 2017; 28: 2801-12.

68. Harre U, Georgess D, Bang H, et al. Induction of osteoclastogenesis and bone loss by human autoantibodies against citrullinated vimentin. J Clin Invest. 2012; 122: 1791-802.

69. Schett G. Autoimmunity as a trigger for structural bone damage in rheumatoid arthritis. Modern Rheumatol. 2017; 27: 193-7.

70. Koppejan H, Trouw LA, Sokolove J, et al. Role of anti-carbamylated protein antibodies compared to anti-citrullinated protein antibodies in indigenous North Americans with rheumatoid arthritis, their first-degree relatives, and healthy controls. Arthritis Rheumatol. 2016; 68: 2090-8.

71. Cutolo M, Kitas GD, van Riel PL. Burden of disease in treated rheumatoid arthritis patients: going beyond the joint. Semin Arthritis Rheum. 2014; 43: 479-88.

72. Coulson KA, Reed G, Gilliam BE, et al. Factors influencing fracture risk, $\mathrm{T}$ score, and management of osteoporosis in patients with rheumatoid arthritis in the Consortium of Rheumatology Researchers of North America (CORRONA) registry. J Clin Rheumatol. 2009; 15: 155-60.

73. Cohen SB, Dore RK, Lane NE, et al. Deno- sumab treatment effects on structural damage, bone mineral density, and bone turnover in rheumatoid arthritis: a twelve month, multicenter, randomized, double-blind, placebocontrolled, phase II clinical trial. Arthritis Rheumatol. 2008; 58: 1299-309.

74. Manara M, Sinigaglia L. Bone and TNF in rheumatoid arthritis: clinical implications. RMD Open. 2015; 1: e000065.

75. Lake F, Proudman S. Rheumatoid arthritis and lung disease: from mechanisms to a practical approach. Semin Respir Crit Care Med. 2014; 35: 222-38.

76. Vij R, Strek ME. Diagnosis and treatment of connective tissue disease-associated interstitial lung disease. Chest. 2013; 143: 814-24.

77. Kobayashi A, Okamoto H. Treatment of interstitial lung diseases associated with connective tissue diseases. Expert Rev Clin Pharmacol. 2012; 5: 219-27.

78. Fischer A, du Bois R. Interstitial lung disease in connective tissue disorders. Lancet. 2012; 380: 689-98.

79. Puechal X, Gottenberg JE, Berthelot JM, et al. Rituximab therapy for systemic vasculitis associated with rheumatoid arthritis: results from the autoimmunity and rituximab registry. Arthritis Care Res. 2012; 64: 331-9.

80. Puechal X, Miceli-Richard C, Mejjad O, et al. Antitumour necrosis factor treatment in patients with refractory systemic vasculitis associated with rheumatoid arthritis. Ann Rheum Dis. 2008; 67: 880-4.

81. Loza E, Lajas C, Andreu JL, et al. Consensus statement on a framework for the management of comorbidity and extra-articular manifestations in rheumatoid arthritis. Rheumatol Int. 2015; 35: 445-58. 